\title{
External ventricular drainage for posthemorrhagic ventricular dilatation in preterm infants: insights on efficacy and failure
}

\author{
Laura C. De Angelis, MD, ${ }^{1,5}$ Alessandro Parodi, MD, 1,5 Marianna Sebastiani, MD,1,5 \\ Alessandro Consales, MD, ${ }^{2}$ Giuseppe M. Ravegnani, MD, ${ }^{2}$ Mariasavina Severino, MD, ${ }^{3}$ \\ Domenico Tortora, MD, PhD, ${ }^{3}$ Andrea Rossi, MD, ${ }^{3,4}$ Mariya Malova, MD, ${ }^{1,5}$ Diego Minghetti, MD, ${ }^{1,5}$ \\ Armando Cama, MD, ${ }^{2,4}$ Gianluca Piatelli, MD, ${ }^{2}$ and Luca A. Ramenghi, MD, PhD ${ }^{1,5}$ \\ 1Department Mother and Child, Neonatal Intensive Care Unit, IRCCS Istituto Giannina Gaslini, Genoa; '2Department of \\ Neurosurgery, IRCCS Istituto Giannina Gaslini, Genoa; ${ }^{2}$ Neuroradiology Unit, IRCCS Istituto Giannina Gaslini, Genoa; \\ ${ }^{4}$ Department of Health Sciences (DISSAL), University of Genoa, Genoa; and ${ }^{5}$ Department of Neurosciences, Rehabilitation, \\ Ophthalmology, Genetics, Maternal and Child Health (DINOGMI), University of Genoa, Genoa, Italy
}

\begin{abstract}
OBJECTIVE The objective of this study was to describe the clinical and neuroradiological characteristics of a cohort of preterm infants who had undergone external ventricular drain insertion as a temporary measure to treat posthemorrhagic ventricular dilatation. In addition, the authors investigated the factors predicting permanent shunt dependency.
\end{abstract}

METHODS The authors retrospectively reviewed the medical records of a cohort of preterm infants who had undergone external ventricular drain insertion at Gaslini Children's Hospital (Genoa, Italy) between March 2012 and February 2018. They also analyzed clinical characteristics and magnetic resonance imaging data, including diffusion- and susceptibilityweighted imaging studies, which were obtained before both catheter insertion and removal.

RESULTS Twenty-eight infants were included in the study. The mean gestational age was $28.2 \pm 2.7$ weeks, and the mean birth weight was $1209 \pm 476 \mathrm{~g}$. A permanent ventriculoperitoneal shunt was inserted in 15/28 (53.6\%) infants because of the failure of external ventricular drainage as a temporary treatment option. Compared with the shunt-free group, the shunt-dependent group had a significantly lower gestational age (29.3 \pm 2.3 vs $27.2 \pm 2.7$ weeks, $p=0.035)$ and tended toward a lower birth weight $(p=0.056)$. None of the clinical and neuroradiological characteristics significantly differed between the shunt-free and shunt-dependent groups at the time of catheter insertion. As expected, ventricular parameters as well as the intraventricular extension of intracerebral hemorrhage, as assessed using the intraventricular hemorrhage score, were reportedly higher in the shunt-dependent group than in the shunt-free group before catheter removal.

CONCLUSIONS External ventricular drainage is a reliable first-line treatment for posthemorrhagic hydrocephalus. However, predicting its efficacy as a unique treatment remains challenging. A lower gestational age is associated with a higher risk of posthemorrhagic hydrocephalus progression, suggesting that the more undeveloped the mechanisms for the clearance of blood degradation products, the greater the risk of requiring permanent cerebrospinal fluid diversion, although sophisticated MRI investigations are currently unable to corroborate this hypothesis.

https://thejns.org/doi/abs/10.3171/2021.5.PEDS20928

KEYWORDS posthemorrhagic hydrocephalus; ventricular dilatation; external ventricular drainage; diffusion-weighted imaging; preterm infants

$\mathrm{P}$ OSTHEMORRHAGIC ventricular dilatation (PHVD) is a significant complication of germinal matrix hemorrhage-intraventricular hemorrhage $(\mathrm{GMH}-\mathrm{IVH})^{1}$ as well as a leading cause of neurodevelopmental impairment in preterm infants. ${ }^{2}$ Approximately $33 \%$ of preterm infants with GMH-IVH subsequently develop PHVD, and its incidence correlates with the severity of IVH. ${ }^{3}$ Although PHVD spontaneously regresses in nearly onethird of cases, cerebrospinal fluid (CSF) drainage is often required, ${ }^{4}$ with a permanent shunt needed in up to $34 \%$

ABBREVIATIONS a-III ventricle width = adapted third ventricle width; $A D=$ anti-displacement; $A D C=$ apparent diffusion coefficient; a-AHW = adapted anterior horn width; $\mathrm{a}-\mathrm{TOD}=$ adapted thalamo-occipital distance; $\mathrm{a}-\mathrm{VI}=$ adapted VI; CSF = cerebrospinal fluid; CUS = cranial ultrasound; DWI = diffusion-weighted imaging; EVD = external ventricular drainage; $\mathrm{GMH}$ = germinal matrix hemorrhage; IVH = intraventricular hemorrhage; MRI = magnetic resonance imaging; $P H V D=$ posthemorrhagic ventricular dilatation; $\mathrm{ROI}$ = region of interest; SWI = susceptibility-weighted imaging; VI = ventricular index; VPS = ventriculoperitoneal shunt.

SUBMITTED November 25, 2020. ACCEPTED May 12, 2021.

INCLUDE WHEN CITING Published online September 3, 2021; DOI: 10.3171/2021.5.PEDS20928. 
of patients. ${ }^{5,6}$ Shunt dependency is associated with several detrimental complications secondary to shunt occlusion, displacement, and infection.? Therefore, temporary PHVD treatment options, including serial lumbar puncture, ventriculosubgaleal shunting, Ommaya reservoir placement, external ventricular drainage (EVD), endoscopic third ventriculostomy, and endoscopic coagulation of the choroid plexus, are often considered. ${ }^{8-10}$

To date, however, the best therapeutic procedure and optimal treatment time have not been established. ${ }^{11}$ At our institution, EVD is the initial treatment of choice for verylow-birth-weight infants with posthemorrhagic hydrocephalus. ${ }^{12-14}$ This procedure is performed once the ventricular index (VI) is greater than the 97th percentile for gestational age $+4 \mathrm{~mm}$, according to Levene..$^{15}$ If the progression of ventricular dilatation is not effectively treated using EVD, a permanent ventriculoperitoneal shunt (VPS) is inserted. In addition to serial ultrasound, brain magnetic resonance imaging (MRI) is routinely performed before both catheter insertion and removal in order to monitor ventricular dilatation and to guide treatment planning and decisions.

In the present study, we retrospectively reviewed the clinical and neuroradiological characteristics of preterm infants with PHVD treated with EVD as well as investigated the predictive factors associated with the need for permanent shunt insertion after EVD failure.

\section{Methods}

Infants with a gestational age $\leq 34$ weeks who had been born at Gaslini Children's Hospital (Genoa, Italy) between March 2012 and February 2018 and who had developed progressive PHVD were identified by retrospectively reviewing electronic medical records. Those who had initially been treated with EVD were included in the study. Infants with prenatal IVH, other known causes of ventricular dilatation, and congenital and chromosomal anomalies were excluded. For every patient, detailed clinical, cranial ultrasound (CUS), and neurosurgical data were retrieved from their clinical charts. Brain MR images were independently reviewed by two neuroradiologists (M. Severino and D. Tortora) blinded to the clinical data. This study was approved by the Institutional Review Board of Gaslini Children's Hospital, Genoa, Italy.

\section{PHVD Diagnosis and Management}

Preterm infants with IVH were studied using serial CUS scans according to the Italian Society of Neonatology guidelines. CUS scanning of the anterior fontanelle was performed by experienced neonatologists using a convex probe (Prosound Alpha 7, Aloka) at a transducer frequency of $8 \mathrm{MHz}$. The severity of GMH-IVH was graded according to the method described by Volpe. ${ }^{16}$ Ventricular dilatation was defined as a VI > 97th percentile for gestational age, according to Levene.$^{15}$ In infants with progressive ventricular dilatation, CUS was performed at least once a day to evaluate the need for neurosurgical intervention.

Our pediatric neurosurgical unit has long-standing experience with EVD as a temporary measure for hydrocephalus and performs nearly 20 interventions every year, both in children and in preterm infants. Within the study period, both symptomatic and asymptomatic infants with a VI exceeding the 97th percentile for gestational age +4 mm underwent brain MRI and were promptly treated with EVD in our neurosurgical department. The procedure was performed regardless of the infant's weight or postmenstrual age. The drainage tube was inserted into the anterior horn of the dilated lateral ventricle and was connected to an external drainage system according to the method outlined below, first described by Andreussi et al. ${ }^{13}$

\section{Ventricular Catheter}

To perform the procedure, a ventricular catheter is associated with an MRI-compatible anti-displacement (AD) device (Ketron Peek 1000, Ricordini Pietro \& C. srl) consisting of a threaded cylinder with a basal plate (Fig. 1A) and a washer fitting the threaded cylinder plate (Fig. 1B).

Directly fixed to the thecal bone, the AD forms a mechanical bridge between the intracranial and extracranial spaces, preventing catheter dislocation and keeping epidural and extracranial spaces divided. Direct contact between the ventricular catheter and skin is thus prevented to reduce the potential risk of infection. This device has been specifically developed for pediatric patients younger than 3 years of age, the hardest to treat among patients requiring EVD. To prevent any CSF leakage, the 2.6-mm inner diameter of the hollow bolt has been designed to precisely fit ventricular catheters with an outer diameter of $3 \mathrm{~mm}$ or more (Fig. 1C). Furthermore, the elastic properties of the catheter itself allow its complete adherence to the internal wall of the screw, reducing the risk of dislodgment. A notch on the top of the screw allows one to knot a suture over the catheter to provide further fixation.

\section{Surgical Technique}

A frontal paramedian L-shaped skin flap is harvested with an incision of about $20 \times 20 \mathrm{~mm}$, either right or left, over Kocher's point (Fig. 2A). The skin flaps are harvested by taking into account the need for externalization of the bolt through the center of the flap. Then, a small rectangular craniectomy is made by means of a high-speed drill. The major diameter of the craniectomy should be approximately $5 \mathrm{~mm}$ smaller than the foot of the bolt.

As the AD device should be implanted over a bone surface, both the incision and the underlying craniectomy must be performed more laterally in newborn infants given the presence of the anterior fontanelle. The dura mater is gently detached with a blunt dissector, coagulated, and incised at the center of the craniectomy (Fig. 2B).

The ventricular catheter is then prepared over its stylet in order to be stretched and narrowed, and it is inserted inside the hollow bolt along with its stylet. Although the length of the catheter below the device can be regulated even after positioning the catheter into the ventricles, we recommend placing the device at the desired level before insertion, thus avoiding further maneuvers when the catheter is positioned. The catheter is introduced into the ventricle using the typical technique (Fig. 2C). The foot of the reversed T-shaped bolt is inserted in the epidural space, passing through the margins of the craniectomy, and gen- 

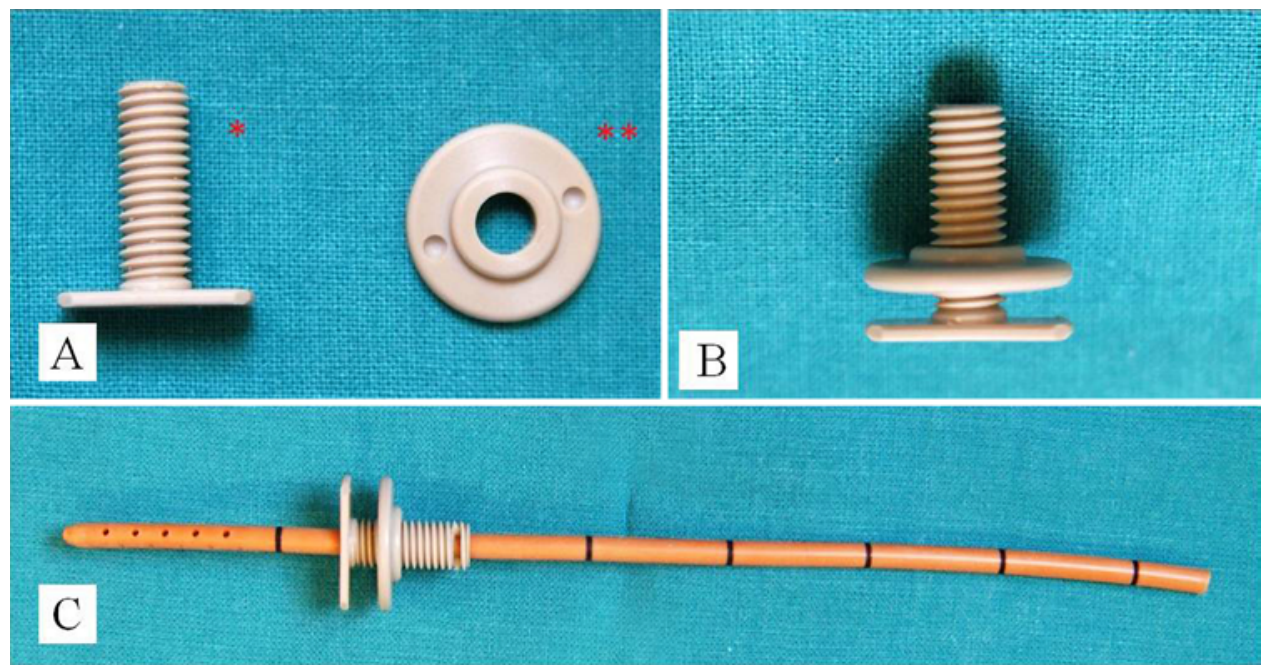

FIG. 1. A: Reversed T-shaped bolt (asterisk) with a long arm made of a hollow threaded cylinder $18 \mathrm{~mm}$ in length with $2.6 \mathrm{~mm}$ of inner diameter and a plate-shaped basal arm (foot) of $15 \times 6 \mathrm{~mm}$. Round washer (double asterisks) $17 \mathrm{~mm}$ in diameter designed to fit the threaded cylinder. B: AD device appearance after insertion of the threaded cylinder in the washer. C: Insertion of the catheter into the $A D$ device.

tly turned $90^{\circ}$ axially, in order to allow both edges of the plate to grip the inner surface of the bone (Fig. 2D).

Once the stylet is withdrawn, the catheter resumes its usual circumference and adheres to the inner side of the hollow bolt, ensuring firm fixation. Subsequently, the AD device is anchored to the dura using two dural sutures. The round washer is then inserted through the threaded bolt and tightened down until the device is securely clamped to the bone (Fig. 2E). At the end of the procedure, the distal part of the bolt comes out from the skin flap through a small incision and a sterile connector links the ventricular catheter to a distal catheter (Fig. 2F).

\section{Postsurgical Management}

To reduce the risk of infection, all patients received 100 $\mathrm{mg} / \mathrm{kg}$ of ceftriaxone immediately before the procedure. In the absence of infection, no additional doses of antibiotics were administered. CSF analysis was performed at the time of surgical intervention and then once a week during the entire duration of the EVD procedure. EVD-related infections were diagnosed in cases in which a positive CSF culture was associated with either clinical signs of central nervous system infection or cytological CSF anomalies.

As the EVD system is valveless and drainage depends only on gravity, the position of the drip cylinder was constantly adjusted to maintain a CSF drainage rate of 10-15 $\mathrm{ml} / \mathrm{kg} /$ day in order to avoid excessive ventricular detention and to maintain the ventricular measures as close to the 97th percentile $+4 \mathrm{~mm}$ as possible. The clinical condition of the infants was closely monitored, and CUS was regularly performed to assess changes in ventricular size as well as to adjust the drainage rate accordingly.

Once the ventricular size stabilized under the 97th percentile $+4 \mathrm{~mm}$, a reabsorption test was performed, wherein the CSF collecting bag was progressively elevated to reduce drain pressure. Functional closure of the drain was thus obtained, and ventricular size was closely monitored using CUS in the following days. In cases in which ventricular size remained stable, a second brain MRI study was performed and the EVD system was removed. In patients with progressive ventricular dilatation or with repeated failed reabsorption tests or after 2 months from external ventricular drain placement, a permanent VPS was considered. This procedure was performed in infants weighing at least 2-2.5 kg, with at least three consecutively negative CSF cultures, and with a CSF protein level below $150 \mathrm{mg} / \mathrm{dl}$, in order to reduce the risk of obstruction.

\section{Brain MRI}

Brain MRI was routinely performed in clinically stable patients before catheter insertion and was repeated before catheter removal, according to our internal protocol. Images were obtained using the 1.5-T MRI system (Intera Achieva, Philips). The infants were moved from the neonatal intensive care unit to the neuroradiology unit and back by a transport incubator provided with a ventilator, pulse oximetry, and infusion pumps. The EVD device was fixed to a mobile stick compatible with high magnetic fields. The examination was performed during natural sleep using the "feed and wrap" technique, and mild sedation with oral midazolam $(0.1 \mu \mathrm{g} / \mathrm{kg})$ was induced to prevent head motion in selected cases, according to the infant's state of arousal and image quality after the first sequence. Hearing protection was provided for all patients. Heart rate and oxygen saturation were monitored by pulse oximetry throughout the examination. Axial, coronal, and sagittal T1- and T2-weighted images were acquired (slice thickness $3.0 \mathrm{~mm}$, no gap). In addition, diffusion-weighted imaging (DWI) sequences were acquired in the axial plane $\left(\mathrm{b}\right.$ value $=1000 \mathrm{~mm}^{2} / \mathrm{sec}$, slice thickness $4.0 \mathrm{~mm}$, no gap). Quantitative evaluation of the apparent diffusion coefficient (ADC) was performed in eight regions of interest (ROIs): frontal, parietooccipital, and parietal white matter and in both cerebellar hemispheres. 


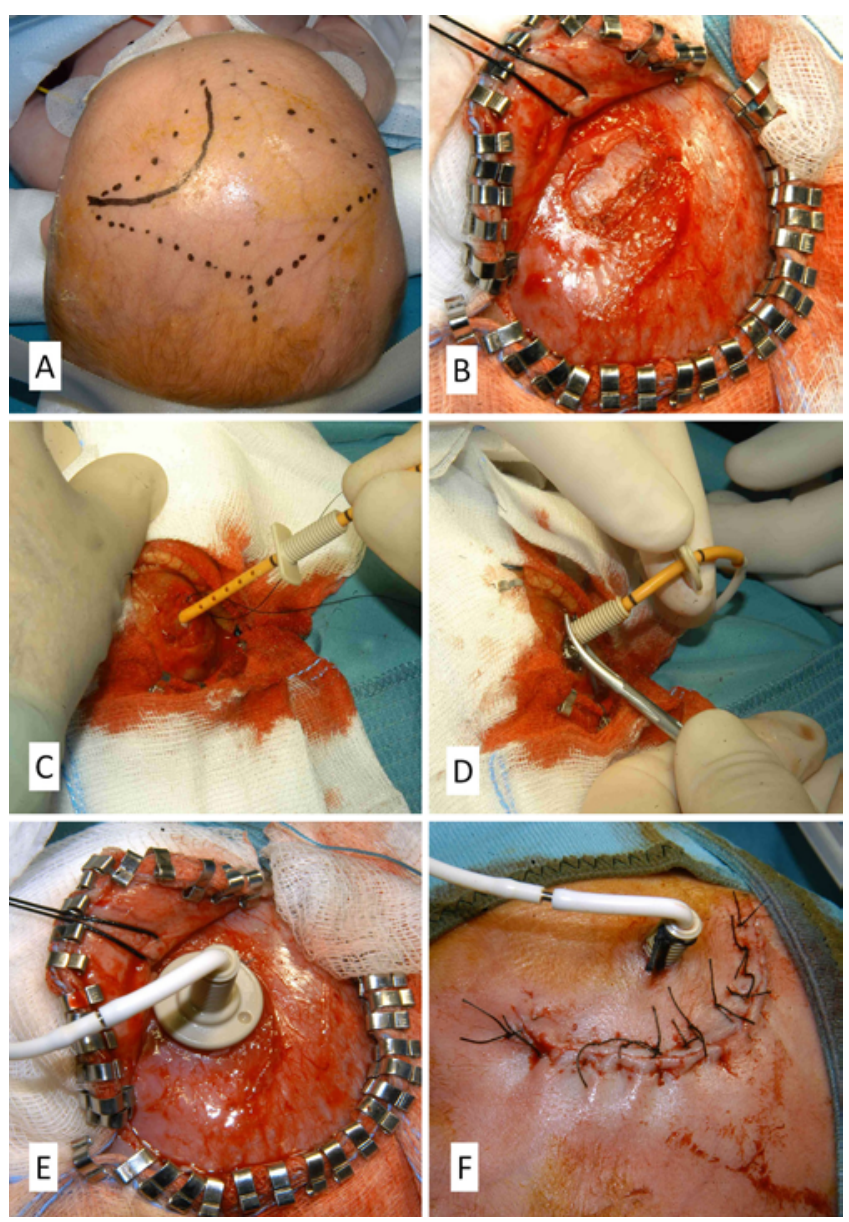

FIG. 2. A: L-shaped skin incision $(20 \times 20 \mathrm{~mm})$ over Kocher's point. B: Small oval craniectomy. C: Insertion of the catheter with the AD device into the lateral ventricle. D: Insertion and $90^{\circ}$ rotation of the device's foot in the epidural space below the thecal bone. E: Insertion and tightening of the washer over the plate and the interposed bone. F: Final appearance of the external ventricular drain emerging from the center of the skin flap.

The following ventricular measurements traditionally used in CUS were adapted to MRI and applied to coronal T2-weighted images: adapted VI (a-VI), adapted anterior horn width (a-AHW), adapted third ventricle width (a-III ventricle width), and adapted thalamo-occipital distance (a-TOD). The presence of cerebral aqueductal stenosis, dilatation of the fourth ventricular outlets, and neuroradiological signs of obstruction of CSF circulation in the cistern of the lamina terminalis, chiasmatic cistern, interpeduncular cistern, pontine cistern, anterior medulla oblongata cistern, cerebellomedullary cistern, and superior cistern was evaluated. The evolution of superficial and ependymal siderosis between the first and second MRI studies was evaluated by analyzing changes in local magnetic field inhomogeneities, resulting in signal loss on susceptibilityweighted imaging (SWI) sequences. In addition, the IVH score was calculated to estimate the severity of IVH. ${ }^{17}$

Statistical analysis was performed using the t-test and chi-square test with SPSS software (IBM Corp.). A p < 0.05 was considered statistically significant.

\section{Results}

A total of 30 preterm infants with a gestational age $\leq 34$ weeks who had developed progressive PHVD were identified within the study period. One patient was excluded because of an antenatal diagnosis of GMH-IVH, and 1 patient died before neurosurgical intervention. The remaining 28 infants were ultimately included in the study. The mean gestational age was $28.2 \pm 2.7$ weeks, and the mean birth weight was $1209 \pm 476 \mathrm{~g}$. A permanent VPS was inserted in 15 of $28(53.6 \%)$ infants because of the failure of temporary EVD. A comparison of clinical characteristics between shunt-free and shunt-dependent groups is shown in Table 1.

Compared with the shunt-free group, the shunt-dependent group had a significantly lower gestational age (29.3 \pm 2.3 vs $27.2 \pm 2.7$ weeks, $\mathrm{p}=0.035)$ and tended toward a lower birth weight $(\mathrm{p}=0.056)$. Patient sex, 1 - and 5-minute Apgar scores, antenatal steroid prophylaxis, type of delivery, inotropic support during the first 72 hours, and prolonged mechanical ventilation did not significantly differ between the two groups. Age at GMH-IVH diagnosis and the presence of bilateral GMH-IVH or tetraventricular hydrocephalus did not differ; however, the shunt-dependent group tended toward a higher frequency of periventricular hemorrhagic infarction ( $73.3 \%$ vs $30.8 \%, \mathrm{p}=0.056)$.

We did not observe significant differences in postmenstrual age at the time of catheter insertion or in CSF protein concentration at the time of drain placement and removal. As expected, both drainage duration $(33.9 \pm 10.9$ vs 25.2 \pm 8.7 days, $p=0.027$ ) and the length of time the catheter remained in place $(52 \pm 20.6$ vs $33.2 \pm 9.2$ days, $p=0.005)$ were significantly longer in the shunt-dependent group than in the shunt-free group. Endoscopic third ventriculostomy was attempted after EVD failure in 9 of $28(32.1 \%)$ infants; however, all of them subsequently required VPS insertion. EVD-related infections were observed in 3 of $28(10.7 \%)$ infants, all belonging to the shunt-dependent group. After EVD insertion, coagulase-negative staphylococci were isolated from the CSF cultures in 2 patients. Both of them were treated with vancomycin and gentamycin. One patient developed methicillin-sensitive Staphylococcus aureus and Klebsiella ventricular abscess, which were treated with meropenem and amikacin. No catheter obstruction or incorrect catheter positioning was reported. No episodes of CSF leakage from the AD device were observed.

All patients included in the study underwent at least one brain MRI study. Brain MRI with DWI and SWI sequences before catheter insertion (MRI 1) were available for 11 of $15(73.3 \%)$ patients in the shunt-dependent group and 11 of $13(84.6 \%)$ patients in the shunt-free group (p $=0.655)$. Brain MRI with DWI and SWI sequences just before catheter removal (MRI 2) were available for all patients. Comparisons of MRI data before catheter insertion and removal are shown in Table 2 .

The first and second MRI scans were obtained at similar postmenstrual ages in the shunt-free and shunt-dependent groups. Before catheter insertion (MRI 1), we found no differences in a-VI, a-AHW, a-III ventricle width, and a-TOD between the two groups. In addition, no significant differences were observed in the frequency of aqueductal 
TABLE 1. Comparison of patient characteristics between shunt-free and shunt-dependent groups

\begin{tabular}{lccc}
\hline \multicolumn{1}{c}{ Variable } & Shunt-Free Group & Shunt-Dependent Group & p Value \\
\hline No. of patients & $13(46.4)$ & $15(53.6)$ & \\
\hline Gestational age in wks & $29.3 \pm 2.3$ & $27.2 \pm 2.7$ & 0.035 \\
\hline Birth weight in g & $1390 \pm 388$ & $1053 \pm 501$ & 0.056 \\
\hline Male sex & $9(69.2)$ & $8(53.3)$ & 0.460 \\
\hline Apgar score at 1 min & $4.5 \pm 2.8$ & $4.7 \pm 2.9$ & 0.837 \\
\hline Apgar score at 5 mins & $7.4 \pm 1.4$ & $7.5 \pm 1.7$ & 0.804 \\
\hline Complete antenatal steroid prophylaxis & $6(46.2)$ & $7(53.8)^{*}$ & $>0.99$ \\
\hline Cesarean section & $6(46.2)$ & $8(53.3)$ & $>0.99$ \\
\hline Inotropic support during first 72 hrs & $5(38.5)$ & $1(7.1) \dagger$ & 0.077 \\
\hline Mechanical ventilation > 7 days & $5(38.5)$ & $9(64.3) \dagger$ & 0.257 \\
\hline Mechanical ventilation > 14 days & $4(30.8)$ & $7(50.0) \dagger$ & 0.440 \\
\hline Age at GMH-IVH diagnosis in days & $1.9 \pm 1.3$ & $2.1 \pm 1.1$ & 0.592 \\
\hline Bilat GMH-IVH & $9(69.2)$ & $13(86.7)$ & 0.372 \\
\hline Periventricular hemorrhagic infarction & $4(30.8)$ & $11(73.3)$ & 0.056 \\
\hline Tetraventricular hydrocephalus & $8(61.5)$ & $11(73.3)$ & 0.689 \\
\hline Time of catheter insertion in days after birth & $19.6 \pm 4.7$ & $23.2 \pm 14.0$ & 0.374 \\
\hline CSF protein at catheter insertion in mg/dl & $187.5 \pm 115.2$ & $160.1 \pm 51.3$ & 0.439 \\
\hline CSF protein at catheter removal in mg/dl & $131.6 \pm 62.9$ & $94.5 \pm 32.7$ & 0.072 \\
\hline Change in CSF protein levels & $-55.9 \pm 107.4$ & $-65.6 \pm 65$ & 0.780 \\
\hline Duration of CSF drainage in days & $25.2 \pm 8.7$ & $33.9 \pm 10.9$ & $\mathbf{0 . 0 2 7}$ \\
\hline Length of time catheter remained in place in days & $33.2 \pm 9.2$ & $52 \pm 20.6$ & $\mathbf{0}$ \\
\hline EVD-related infection & 0 & $3(20.0)$ & 0.226 \\
\hline ETV & 0 & $9(60.0)$ & 0.001 \\
\hline
\end{tabular}

ETV = endoscopic third ventriculostomy.

Data are presented as number (\%) or mean \pm standard deviation, unless indicated otherwise. Boldface type indicates statistical significance.

* Data available for 13 patients.

$\uparrow$ Data available for 14 patients.

stenosis, dilatation of fourth ventricular outlets, and obstruction of CSF spaces (Table 2).

Brain MRI before catheter removal revealed significantly higher values of a-AHW $(13.2 \pm 4.2$ vs $9.1 \pm 5.9$ $\mathrm{mm}, \mathrm{p}=0.049)$ and a-III ventricle width $(8.2 \pm 4.1$ vs 4.4 $\pm 1.7 \mathrm{~mm}, \mathrm{p}=0.004$ ) in the shunt-dependent group than in the shunt-free group. No differences were observed for a-VI and a-TOD between the two groups. None of the patients in the shunt-free group and 6/15 (40\%) patients in the shunt-dependent group presented with aqueductal stenosis $(\mathrm{p}=0.018)$, and there was a tendency toward a greater frequency of dilatation of fourth ventricular outlets in the shunt-dependent group than in the shunt-free group $(53.3 \%$ vs $15.4 \%, \mathrm{p}=0.055)$.

The IVH score was significantly higher on the second brain MRI study $(6.7 \pm 5.5$ vs $2.1 \pm 2.3, \mathrm{p}=0.008)$ and the difference in IVH scores between the first and second MRI studies was lower $(7.1 \pm 3.7$ vs $10.9 \pm 4.7$, p = 0.028 ) in the shunt-dependent group than in the shunt-free group. We found no differences between the two groups in terms of the evolution of obstruction of CSF spaces. In addition, the evolution of cortical cerebral, cerebellar, and ependymal siderosis on SWI sequences between the two MRI studies did not significantly differ between the two groups. The mean ADC values calculated for each ROI did not significantly differ between the two MRI studies between the two groups; however, in the shunt-free group, a nonsignificant tendency for higher ADC values was observed in the frontal white matter on the second MRI study (Table 3).

\section{Discussion}

In the present study, we describe our experience using EVD as the initial treatment of choice for infants with PHVD and compare the clinical and neuroradiological characteristics of a group of preterm infants who were successfully treated with temporary EVD with those of a group that required permanent CSF diversion after EVD failure. Our data show that 13 of 28 (46.4\%) infants were effectively treated with EVD performed when the VI exceeded the 97th percentile for gestational age $+4 \mathrm{~mm}$. Our findings are consistent with those of a recent metaanalysis of preterm infants that revealed that the resolution of hydrocephalus was higher with EVD (31.8\%) than with ventricular access devices (17.5\%) or ventriculosubgaleal shunts $(13.9 \%) .{ }^{11}$

At present, there is no clear consensus regarding the 
TABLE 2. Comparison of brain MRI data between shunt-free and shunt-dependent groups

\begin{tabular}{|c|c|c|c|}
\hline Variable & Shunt-Free Group & Shunt-Dependent Group & $p$ Value \\
\hline \multicolumn{4}{|l|}{ MRI 1} \\
\hline Available brain MRI scans & $11 / 13(84.6)$ & $11 / 15(73.3)$ & 0.655 \\
\hline Aqueductal stenosis & $1(9.1)$ & $3(27.3)$ & 0.587 \\
\hline Dilatation of 4 th ventricular outlets & $6(54.5)$ & $5(45.5)$ & $>0.99$ \\
\hline Basal cistern obstruction & $6(54.5)$ & $5(45.5)$ & $>0.99$ \\
\hline Perimesencephalic obstruction & $5(45.5)$ & $6(54.5)$ & $>0.99$ \\
\hline Prepontine obstruction & $8(72.7)$ & $7(63.6)$ & $>0.99$ \\
\hline Peribulbar obstruction & $9(81.8)$ & $8(72.7)$ & $>0.99$ \\
\hline Pericerebellar obstruction & $10(90.9)$ & $11(100.0)$ & $>0.99$ \\
\hline Postmenstrual age at scan in wks & $32.0 \pm 2.1$ & $31.1 \pm 3.8$ & 0.495 \\
\hline $\mathrm{a}-\mathrm{Vl}$ in $\mathrm{mm}$ & $18.7 \pm 3.1$ & $17.7 \pm 2.1$ & 0.336 \\
\hline a-AHW in mm & $16.0 \pm 4.6$ & $16.4 \pm 3.2$ & 0.830 \\
\hline a-III ventricle width in $\mathrm{mm}$ & $8.9 \pm 2.9$ & $9.3 \pm 2.8$ & 0.755 \\
\hline a-TOD in mm & $31.9 \pm 7.8$ & $30.0 \pm 8.0$ & 0.547 \\
\hline IVH score & $12 \pm 4.4$ & $13.8 \pm 4.8$ & 0.312 \\
\hline \multicolumn{4}{|l|}{ MRI 2} \\
\hline Available brain MRI scans & $13 / 13$ & $15 / 15$ & \\
\hline Aqueductal stenosis & $0(0)$ & $6(40.0)$ & 0.018 \\
\hline Dilatation of 4 th ventricular outlets & $2(15.4)$ & $8(53.3)$ & 0.055 \\
\hline Postmenstrual age at scan in wks & $37.3 \pm 2.2$ & $36.0 \pm 3.6$ & 0.252 \\
\hline $\mathrm{a}-\mathrm{VI}$ in $\mathrm{mm}$ & $16.1 \pm 4.2$ & $18.2 \pm 4.0$ & 0.196 \\
\hline a-AHW in mm & $9.1 \pm 5.9$ & $13.2 \pm 4.2$ & 0.049 \\
\hline a-III ventricle width in $\mathrm{mm}$ & $4.4 \pm 1.7$ & $8.2 \pm 4.1$ & 0.004 \\
\hline a-TOD in mm & $25.8 \pm 9.1$ & $27.1 \pm 7.3$ & 0.687 \\
\hline IVH score & $2.1 \pm 2.3$ & $6.7 \pm 5.5$ & 0.008 \\
\hline Change in IVH score & $10.9 \pm 4.7$ & $7.1 \pm 3.7$ & 0.028 \\
\hline
\end{tabular}

Data are presented as number (\%) or mean \pm standard deviation, unless indicated otherwise. Boldface type indicates statistical significance.

optimal timing of neurosurgical intervention in preterm infants with PHVD, although the majority of centers adopt a VI cutoff value of the 97th percentile for gestational age $+4 \mathrm{~mm} .{ }^{18} \mathrm{~A}$ recent randomized controlled trial conducted by de Vries et al. compared this approach (VI > 97th percentile $+4 \mathrm{~mm}$ ) with a more compelling cutoff value (VI > 97th percentile) and reassuringly showed a reduced number of interventions, including VPS placement, in the 97th percentile $+4 \mathrm{~mm}$ arm. ${ }^{19}$ Nevertheless, a more recent MRI subgroup analysis of the same study population showed a more severe loss of white matter in infants treated according to the cutoff value of $\mathrm{VI}>97$ th percentile $+4 \mathrm{~mm} \cdot{ }^{20} \mathrm{In}$ our study, all patients were treated once the 97th percentile $+4 \mathrm{~mm}$ line was exceeded; therefore, we were not able to assess which VI cutoff might be preferable. However, we did not find a significant difference in mean postmenstrual age at the time of EVD between the shunt-dependent and shunt-free groups.

In our cohort, the length of time the drain remained in place was significantly longer in infants who eventually underwent VPS insertion. Although there are no unequivocal indications for temporary EVD failure, the amount of time the catheter remains in place depends on a bal- ance between the risk of infection and the time required for reabsorption mechanisms to mature. ${ }^{21}$ Besides the VI, other ventricular measures, such as AHW, third ventricle width, and TOD, are useful additional parameters to assess PHVD progression. ${ }^{22,23}$ While an understandable significant difference between the shunt-free and shuntdependent groups was observed in a-AHW and a-III ventricle width on the second MRI study given the failure of CSF reabsorption, these parameters were not predictive of shunt dependency.

Although EVD is only one possible temporary measure to treat posthemorrhagic hydrocephalus, it has several advantages. Contrary to the techniques requiring several taps, EVD allows continuous CSF drainage, mimicking a more physiological CSF reabsorption. In addition, the contemporary removal of IVH-related blood products, debris, and inflammatory cytokines from CSF spaces may accelerate the resolution of CSF obstruction. ${ }^{6}$ Moreover, CSF drainage pressure can be easily and rapidly adjusted according to clinical parameters and degree of ventricular dilatation. Nevertheless, EVD requires frequent neurosurgical assessments to evaluate individual CSF drainage requirements as well as regular CUS monitoring to avoid 
TABLE 3. ADC values in eight ROls in the shunt-free and shunt-dependent groups

\begin{tabular}{|c|c|c|c|}
\hline ROI & Shunt-Free Group & Shunt-Dependent Group & $\mathrm{p}$ Value \\
\hline \multicolumn{4}{|l|}{ MRI 1} \\
\hline \multicolumn{4}{|c|}{ Frontal white matter } \\
\hline $\mathrm{Rt}$ & $1854.3 \pm 348.4$ & $1777.4 \pm 308.1$ & 0.526 \\
\hline $\mathrm{Lt}$ & $1857.1 \pm 275.0$ & $1702.4 \pm 227.5$ & 0.167 \\
\hline \multicolumn{4}{|c|}{ Parietooccipital white matter } \\
\hline Rt & $1760.3 \pm 260.0$ & $1676.8 \pm 139.1$ & 0.362 \\
\hline $\mathrm{Lt}$ & $1857.0 \pm 376.2$ & $1802.6 \pm 117.0$ & 0.655 \\
\hline \multicolumn{4}{|c|}{ Parietal white matter } \\
\hline $\mathrm{Rt}$ & $2027.3 \pm 567.8$ & $2033.6 \pm 383.5$ & 0.976 \\
\hline $\mathrm{Lt}$ & $2036.1 \pm 503.2$ & $2082.1 \pm 472.4$ & 0.827 \\
\hline \multicolumn{4}{|l|}{ Cerebellum } \\
\hline Rt & $1275.7 \pm 241.7$ & $1268.4 \pm 149.6$ & 0.933 \\
\hline Lt & $1294.0 \pm 227.4$ & $1268.4 \pm 168.1$ & 0.767 \\
\hline \multicolumn{4}{|l|}{ MRI $2^{*}$} \\
\hline \multicolumn{4}{|c|}{ Frontal white matter } \\
\hline $\mathrm{Rt}$ & $1815.4 \pm 272.4$ & $1692.1 \pm 182.3$ & 0.195 \\
\hline $\mathrm{Lt}$ & $1765.3 \pm 283.4$ & $1593.3 \pm 187.6$ & 0.087 \\
\hline \multicolumn{4}{|c|}{ Parietooccipital white matter } \\
\hline Rt & $1607.3 \pm 338.3$ & $1597.5 \pm 277.7$ & 0.937 \\
\hline $\mathrm{Lt}$ & $1776.7 \pm 371.3$ & $1563.8 \pm 276.1$ & 0.114 \\
\hline \multicolumn{4}{|c|}{ Parietal white matter } \\
\hline $\mathrm{Rt}$ & $1762.4 \pm 345.0$ & $1746.1 \pm 292.9$ & 0.897 \\
\hline Lt & $1789.8 \pm 294.3$ & $1687.3 \pm 321.4$ & 0.401 \\
\hline \multicolumn{4}{|l|}{ Cerebellum } \\
\hline Rt & $1219.4 \pm 169.7$ & $1241.7 \pm 162.2$ & 0.732 \\
\hline $\mathrm{Lt}$ & $1225.5 \pm 182.6$ & $1238.0 \pm 151.3$ & 0.851 \\
\hline
\end{tabular}

the potential risk of overdrainage. Furthermore, EVD is associated with a relevant risk of infection, with reported incidence rates of $0 \%-11 \%$ across studies. ${ }^{11}$ In our cohort, EVD-related infections were observed in 3 of $28(10.7 \%)$ infants, comparable to data from the literature.

We found a significant association between lower gestational age and the need for a permanent shunt. This finding is consistent with both a higher risk of severe IVH at an earlier gestational age ${ }^{24,25}$ and the remarkable vulnerability of CSF drainage systems in more prematurely born infants. ${ }^{1}$

The severity of IVH is one of the factors predicting adverse short-term outcomes in patients with PHVD. ${ }^{5}$ In our study, age at IVH diagnosis and the presence of bilateral IVH or tetraventricular hydrocephalus did not differ between the two groups. Simultaneously, we observed a tendency toward a higher frequency of periventricular hemorrhagic infarction in the shunt-dependent group than in the shunt-free group; the lack of a significant difference between the two groups may be attributable to our small sample size. We also analyzed several clinical characteristics associated with IVH development, ${ }^{26}$ including sex, 1- and 5-minute Apgar scores, antenatal steroid prophylaxis, type of delivery, inotropic support during the first 72 hours, and prolonged mechanical ventilation; however, none of them had an effect on the subsequent risk of shunt dependency. We speculate that the main risk factors for IVH pathogenesis could lose their importance in predicting PHVD progression probably because of the different pathogeneses of CSF reabsorption failure.

PHVD is a common complication of GMH-IVH and is characterized by cerebroventricular expansion, eventually resulting in compression of the surrounding brain parenchyma. ${ }^{27}$ Subsequent brain injury worsens the neurodevelopmental prognosis of infants with GMH-IVH. Although PHVD pathogenesis is complex and remains largely unexplored, a number of plausible mechanisms have been hypothesized. ${ }^{28}$ Blood debris secondary to IVH may obstruct the interventricular foramina and impair CSF circulation even if fibrinolytic therapy fails to reduce PHVD incidence and outcomes. ${ }^{29}$ Accumulating evidence suggests the role of hemoglobin metabolites and iron, which penetrate into the brain parenchyma after GMH-IVH and interfere with the dynamics of CSF production, although the precise pathogenetic mechanism remains unclear. ${ }^{30-32}$ In addition, inflammatory alterations following GMH-IVH, including subependymal gliosis, fibrosing arachnoiditis, and meningeal fibrosis, may alter barrier dynamics in the microvasculature and ependymal lining, impairing CSF-interstitial fluid exchange. ${ }^{33-35}$

In the present study, the shunt-dependent group exhibited a significantly higher frequency of aqueductal stenosis 
on MRI performed before catheter removal and showed a nonsignificant trend toward a higher frequency of dilatation of the fourth ventricular outlets than the shunt-free group. On the other hand, we did not find significant differences in the evolution of the obstruction of CSF spaces or in that of cortical ependymal and cerebellar siderosis on first and second MRI studies between the two groups.

As previously discussed, one of the benefits of EVD is the continuous removal of CSF rich in protein and blood degradation products. ${ }^{1}$ In our work, no significant differences were observed in CSF protein concentration at the time of catheter insertion and removal between the two groups. Simultaneously, the IVH score, which indicates the amount of blood and blood degradation products inside the ventricular system, was significantly higher in the shunt-dependent group than in the shunt-free group on the second MRI study. Our data suggest that a failure in removing blood degradation products is associated with a higher risk of shunt dependency.

In a further attempt to avoid VPS insertion, endoscopic third ventriculostomy was performed in 9 patients after EVD failure; however, all of these patients eventually required permanent CSF diversion. Although this procedure is reportedly effective in young infants with aqueductal stenosis, preterm infants with PHVD seem to not benefit from this neurosurgical procedure. ${ }^{36}$

From a neuroradiological point of view, quantitative ADC evaluation on DWI can be used to assess the overall health of the white matter; however, data regarding the correlation between ADC and brain injury or neurodevelopmental outcomes remain controversial. ${ }^{23}$ In the present study, we observed a nonsignificant trend toward higher ADC values in the frontal white matter on the second MRI study in the shunt-free group. We hypothesize that this finding is associated with an increase in the size of the extracellular water compartment resulting from more effective transependymal CSF absorption. However, further studies are warranted to verify this hypothesis.

To our knowledge, this is one of the largest cohort studies on preterm infants with PHVD who were temporarily treated with EVD as well as the first study to compare the neuroradiological parameters before and immediately after EVD to explore the possible predictive factors associated with shunt dependency. Nevertheless, as PHVD requiring intervention is a relatively uncommon condition, the statistical power of our analysis is limited. In our cohort, gestational age was the only preprocedural parameter associated with a higher risk of EVD failure. As expected, infants with shunt dependency showed higher ventricular measures, specifically AHW and third ventricle width, before catheter removal. In addition, they showed greater intraventricular extension of intracerebral hemorrhage, as estimated using the IVH score.

\section{Conclusions}

Our data suggest that EVD is a reliable first treatment option for infants with PHVD, although the prediction of its efficacy as a definitive treatment option remains challenging. A lower gestational age is associated with a higher risk of PHVD progression, suggesting that the more unde- veloped the mechanism for the clearance of blood degradation products, the greater the risk of requiring permanent CSF diversion, although sophisticated MRI investigations are currently unable to corroborate this hypothesis.

\section{References}

1. Robinson S. Neonatal posthemorrhagic hydrocephalus from prematurity: pathophysiology and current treatment concepts. J Neurosurg Pediatr. 2012;9(3):242-258.

2. Futagi Y, Suzuki Y, Toribe Y, Nakano H, Morimoto K. Neurodevelopmental outcome in children with posthemorrhagic hydrocephalus. Pediatr Neurol. 2005;33(1):26-32.

3. Cherian S, Whitelaw A, Thoresen M, Love S. The pathogenesis of neonatal post-hemorrhagic hydrocephalus. Brain Pathol. 2004;14(3):305-311.

4. Ellenbogen JR, Waqar M, Pettorini B. Management of posthaemorrhagic hydrocephalus in premature infants. J Clin Neurosci. 2016;31:30-34.

5. Murphy BP, Inder TE, Rooks V, Taylor GA, Anderson NJ, et al. Posthaemorrhagic ventricular dilatation in the premature infant: natural history and predictors of outcome. Arch Dis Child Fetal Neonatal Ed. 2002;87(1):F37-F41.

6. Whitelaw A, Aquilina K. Management of posthaemorrhagic ventricular dilatation. Arch Dis Child Fetal Neonatal Ed. 2012;97(3):F229-F233.

7. Diwakar K, Hader WJ, Soraisham A, Amin H, Tang S, et al. Long-term neurodevelopmental and growth outcomes of premature infants born at $<29$ week gestational age with post-hemorrhagic hydrocephalus treated with ventriculoperitoneal shunt. Indian J Pediatr. 2017;84(9):662-669.

8. Berger A, Weninger M, Reinprecht A, Haschke N, Kohlhauser C, Pollak A. Long-term experience with subcutaneously tunneled external ventricular drainage in preterm infants. Childs Nerv Syst. 2000;16(2):103-110.

9. Zaben M, Finnigan A, Bhatti MI, Leach P. The initial neurosurgical interventions for the treatment of posthaemorrhagic hydrocephalus in preterm infants: a focused review. $\mathrm{Br} \mathrm{J}$ Neurosurg. 2016;30(1):7-10.

10. Tröbs RB, Sander V. Posthemorrhagic hydrocephalus in extremely low birth weight infants: Ommaya reservoir vs. ventriculoperitoneal shunt. Childs Nerv Syst. 2015;31(8):12611266.

11. Badhiwala JH, Hong CJ, Nassiri F, Hong BY, Riva-Cambrin J, Kulkarni AV. Treatment of posthemorrhagic ventricular dilation in preterm infants: a systematic review and metaanalysis of outcomes and complications. J Neurosurg Pediatr. 2015;16(5):545-555.

12. Massone ML, Cama A, Puccio VF, Montobbio G, Soliani M, et al. Management of post-hemorrhagic ventricular dilation in premature infants with long-term external ventricular drainage. Eur J Pediatr Surg. 1993;3(1)(suppl 1):22-23.

13. Andreussi L, Carini S, Cama A, Jannuzzi C, Mantero E. A durable system for external ventricular drainage: description of the device, operative technique and results of CSF shunt colonisation. Eur J Pediatr Surg. 1993;3(1)(suppl 1):21-22.

14. Andreussi L, Cama A, Carini S, Jannuzzi C. Long-term external shunting of cerebrospinal fluid. Technique, indications and results. Article in Italian. Minerva Pediatr. 1992;44(10 Suppl 1):59-63.

15. Levene MI. Measurement of the growth of the lateral ventricle in preterm infants with real-time ultrasound. Arch Dis Child. 1981;56:905-910.

16. Volpe JJ. Neurology of the newborn. Major Probl Clin Pediatr. 1981;22:1-648.

17. Hallevi H, Dar NS, Barreto AD, Morales MM, Martin-Schild $\mathrm{S}$, et al. The IVH score: a novel tool for estimating intraventricular hemorrhage volume: clinical and research implications. Crit Care Med. 2009;37(3):969-974, e1. 
18. Whitelaw A, Evans D, Carter M, Thoresen M, Wroblewska J, et al. Randomized clinical trial of prevention of hydrocephalus after intraventricular hemorrhage in preterm infants: brain-washing versus tapping fluid. Pediatrics. 2007;119(5): e1071-e1078.

19. de Vries LS, Groenendaal F, Liem KD, Heep A, Brouwer AJ, et al. Treatment thresholds for intervention in posthaemorrhagic ventricular dilation: a randomised controlled trial. Arch Dis Child Fetal Neonatal Ed. 2019;104(1):F70-F75.

20. Cizmeci MN, Khalili N, Claessens NHP, Groenendaal F, Liem KD, et al. Assessment of brain injury and brain volumes after posthemorrhagic ventricular dilatation: a nested substudy of the randomized controlled ELVIS trial. J Pediatr. 2019;208:191-197.e2.

21. Mazzola CA, Choudhri AF, Auguste KI, Limbrick DD Jr, Rogido M, et al. Pediatric hydrocephalus: systematic literature review and evidence-based guidelines. Part 2: Management of posthemorrhagic hydrocephalus in premature infants. J Neurosurg Pediatr. 2014;14(1)(suppl 1):8-23.

22. Davies MW, Swaminathan M, Chuang SL, Betheras FR. Reference ranges for the linear dimensions of the intracrania ventricles in preterm neonates. Arch Dis Child Fetal Neonatal Ed. 2000;82(3):F218-F223.

23. Brouwer MJ, de Vries LS, Groenendaal F, Koopman C, Pistorius LR, et al. New reference values for the neonatal cerebral ventricles. Radiology. 2012;262(1):224-233.

24. Sannia A, Natalizia AR, Parodi A, Malova M, Fumagalli M, et al. Different gestational ages and changing vulnerability of the premature brain. J Matern Fetal Neonatal Med. 2015; 28(1)(suppl 1):2268-2272.

25. Kazan S, Güra A, Uçar T, Korkmaz E, Ongun H, Akyuz M. Hydrocephalus after intraventricular hemorrhage in preterm and low-birth weight infants: analysis of associated risk factors for ventriculoperitoneal shunting. Surg Neurol. 2005; 64(2)(suppl 2):S77-S81.

26. Ment LR, Ådén U, Bauer CR, Bada HS, Carlo WA, et al. Genes and environment in neonatal intraventricular hemorrhage. Semin Perinatol. 2015;39(8):592-603.

27. Jary S, De Carli A, Ramenghi LA, Whitelaw A. Impaired brain growth and neurodevelopment in preterm infants with posthaemorrhagic ventricular dilatation. Acta Paediatr. 2012; 101(7):743-748.

28. Klebe D, McBride D, Krafft PR, Flores JJ, Tang J, Zhang JH. Posthemorrhagic hydrocephalus development after germinal matrix hemorrhage: Established mechanisms and proposed pathways. J Neurosci Res. 2020;98(1):105-120.

29. Strahle J, Garton HJ, Maher CO, Muraszko KM, Keep RF, Xi G. Mechanisms of hydrocephalus after neonatal and adult intraventricular hemorrhage. Transl Stroke Res. 2012;3(1)(suppl 1):25-38.

30. Chen Z, Gao C, Hua Y, Keep RF, Muraszko K, Xi G. Role of iron in brain injury after intraventricular hemorrhage. Stroke. 2011;42(2):465-470.
31. Tortora D, Severino M, Sedlacik J, Toselli B, Malova M, et al. Quantitative susceptibility map analysis in preterm neonates with germinal matrix-intraventricular hemorrhage. J Magn Reson Imaging. 2018;48(5):1199-1207.

32. Tortora D, Martinetti C, Severino M, Uccella S, Malova M, et al. The effects of mild germinal matrix-intraventricular haemorrhage on the developmental white matter microstructure of preterm neonates: a DTI study. Eur Radiol. 2018; 28(3):1157-1166.

33. Karimy JK, Zhang J, Kurland DB, Theriault BC, Duran D, et al. Inflammation-dependent cerebrospinal fluid hypersecretion by the choroid plexus epithelium in posthemorrhagic hydrocephalus. Nat Med. 2017;23(8):997-1003.

34. Chen Q, Feng Z, Tan Q, Guo J, Tang J, et al. Post-hemorrhagic hydrocephalus: Recent advances and new therapeutic insights. J Neurol Sci. 2017;375:220-230.

35. Aquilina K, Chakkarapani E, Thoresen M. Early deterioration of cerebrospinal fluid dynamics in a neonatal piglet model of intraventricular hemorrhage and posthemorrhagic ventricular dilation. J Neurosurg Pediatr. 2012;10(6):529-537.

36. Elgamal EA, El-Dawlatly AA, Murshid WR, El-Watidy SM, Jamjoom ZA. Endoscopic third ventriculostomy for hydrocephalus in children younger than 1 year of age. Childs Nerv Syst. 2011;27(1):111-116.

\section{Disclosures}

The authors report no conflict of interest concerning the materials or methods used in this study or the findings specified in this paper.

\section{Author Contributions}

Conception and design: Parodi, Consales, Ravegnani, Piatelli, Ramenghi. Acquisition of data: Parodi, Sebastiani, Severino, Malova. Analysis and interpretation of data: De Angelis, Consales, Severino, Tortora, Malova. Drafting the article: De Angelis, Sebastiani, Malova. Critically revising the article: De Angelis, Consales, Ravegnani, Rossi, Minghetti, Cama, Piatelli, Ramenghi. Reviewed submitted version of manuscript: De Angelis, Parodi, Sebastiani, Consales, Ravegnani, Severino, Tortora, Rossi, Malova, Minghetti, Piatelli, Ramenghi. Approved the final version of the manuscript on behalf of all authors: De Angelis. Statistical analysis: Severino, Tortora. Administrative/ technical/material support: Rossi, Cama. Study supervision: Piatelli, Ramenghi.

\section{Correspondence}

Laura C. De Angelis: Giannina Gaslini Institute, Genoa, Italy. lallade@gmail.com. 